\title{
Beam profile monitoring at the test beam line at the Compact Linear Collider test facility
}

\author{
M. Olvegård, ${ }^{1,2, *}$ E. Adli, ${ }^{1,3}$ W. Andreazza, ${ }^{1}$ B. Bolzon, ${ }^{1}$ E. Bravin, ${ }^{1}$ N. Chritin,,${ }^{1}$ A. Dabrowski, ${ }^{1}$ \\ S. Döbert, ${ }^{1}$ M. Duraffourg, ${ }^{1}$ T. Lefèvre,,${ }^{1}$ R. Lillestøl, ${ }^{1,3}$ and V. Ziemann ${ }^{2}$ \\ ${ }^{1}$ CERN, European Organization of Nuclear Research, 1211 Genève 23, Switzerland \\ ${ }^{2}$ Uppsala University, Department of Physics and Astronomy, Box 516, 75120 Uppsala, Sweden \\ ${ }^{3}$ University of Oslo, Boks 1072 Blindern, 0316 Oslo, Norway \\ (Received 30 March 2012; revised manuscript received 18 January 2013; published 27 August 2013)
}

\begin{abstract}
The Compact Linear Collider (CLIC) is a study for a future linear electron-positron collider based on a two-beam acceleration scheme in which a high-intensity drive beam is decelerated in order to provide the power to accelerate the main beam for collision in the $\mathrm{TeV}$ range. The power extracted from the drive beam deteriorates the beam quality and increases the energy spread significantly. Monitoring of the beam properties is therefore challenging but essential. These challenges are being addressed experimentally at the CLIC test facility where up to $55 \%$ of the power is extracted from the beam in the test beam line, a small-scale version of the CLIC drive-beam decelerator, leaving the beam with a very wide energy profile. For monitoring of the transverse beam profile and Twiss parameters we use optical transition radiation screens and quadrupole scans. The intra-pulse-train energy spectrum before and after deceleration is measured with segmented beam dumps. In this paper we discuss the performance of these diagnostic devices with a particular emphasis on the large energy spread and its effect on the beam imaging techniques, and with a final outlook to the CLIC drive-beam diagnostics.
\end{abstract}

DOI: 10.1103/PhysRevSTAB.16.082802

PACS numbers: 41.85.Qg, 29.27.Fh, 29.30.Aj, 29.20.Ej

\section{INTRODUCTION}

A promising candidate for performing precision measurements in the multi- $\mathrm{TeV}$ regime is the compact linear collider CLIC [1]. It is based on the two-beam acceleration scheme in which a suitably prepared low-energy, but highintensity drive beam is utilized to distribute the power along the linear accelerator. Next to the accelerating structures, that will operate at a gradient of $100 \mathrm{MeV} / \mathrm{m}$ in order to increase the energy of the main beam to the $\mathrm{TeV}$ range in the $21 \mathrm{~km}$ linac, the power is extracted from the in parallel running drive beam by so-called power extraction and transfer structures (PETS) [2]. Each PETS structure will produce $140 \mathrm{MW}$ of power from the nominal drivebeam current of $101 \mathrm{~A}$. The PETS are arranged in sectors with 1491 PETS in a series that will extract up to $90 \%$ of the beam energy and convert it to $12 \mathrm{GHz}$ radio-frequency (rf) power. Reducing the energy by such a large amount poses a severe challenge to the beam stability $[3,4]$, especially in the light of transporting the beam along the $880-\mathrm{m}$ long decelerators.

The energy distribution at the end of the decelerator can be simulated in PLACET [5,6]. Because of the filling time of the PETS, there will be a high-energy transient followed by a long steady state. The large energy spread and the

\footnotetext{
*maja.olvegard@physics.uu.se
}

Published by the American Physical Society under the terms of the Creative Commons Attribution 3.0 License. Further distribution of this work must maintain attribution to the author(s) and the published article's title, journal citation, and DOI. asymmetric energy distribution is illustrated in the energy histogram in Fig. 7 in Ref. [7]. The peak at the most decelerated particles at about $240 \mathrm{MeV}$, which is $10 \%$ of the initial energy, is accompanied by a substantial highenergy tail up to about $1 \mathrm{GeV}$ with smaller contributions all the way to the initial energy of $2.4 \mathrm{GeV}$. A beam with such an energy profile is a particular challenge for beam monitors, both in terms of energy acceptance and in terms of how different techniques respond to a beam with a large energy spread. Additionally, the high beam intensity poses a threat to the integrity of the beam diagnostic equipment.

These challenges need to be investigated and verified experimentally, which is the purpose of the test beam line (TBL), that is part of the CLIC Test Facility (CTF3) [8] at CERN. In CTF3 a high-intensity beam with a current of $4 \mathrm{~A}$ and a pulse duration of about $1.2 \mu$ s operated at a bunch repetition rate of $1.5 \mathrm{GHz}$ is interleaved with a delay loop and a combiner ring to result in a nearly $30 \mathrm{~A}$ and $140 \mathrm{~ns}$ pulse with a bunch repetition rate of $12 \mathrm{GHz}$. This beam is subsequently guided to the TBL and passed through a sequence of 8 focusing-defocusing cells with space for up to 16 PETS of which 13 are installed so far; see sketch in Fig. 1. The beam current in TBL is about 4 times lower than in CLIC, and in order to compensate for this and to reach the required power level of $140 \mathrm{MW}$ the PETS are 4 times longer than those designed for CLIC. This causes a deceleration of the beam by $5.2 \mathrm{MeV}$ in each PETS. With all PETS installed this means a deceleration from 150 to $67 \mathrm{MeV}$, i.e., extraction of $55 \%$ of the beam energy. We refer to Table I for a juxtaposition of beam parameters for CLIC and TBL. The energy distribution at 

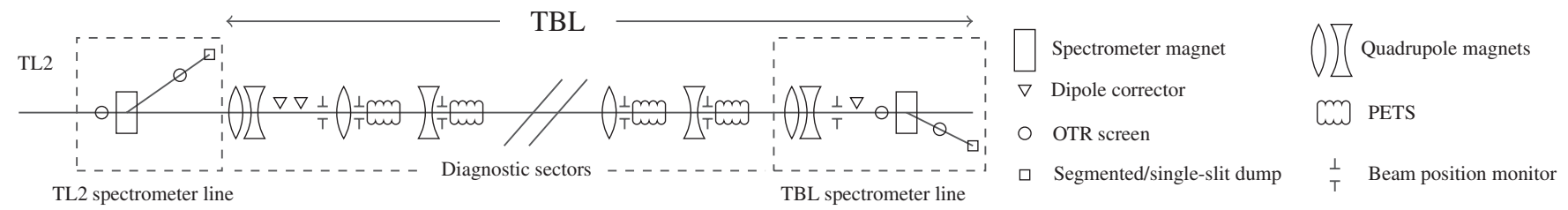

FIG. 1. The layout of TBL showing the locations of the two diagnostic sectors including the TL2 and the TBL spectrometer lines. In its final configuration TBL will contain 16 PETS structures, of which only 4 are shown in the sketch.

the end of the TBL is also simulated with PLACET. Similar to what is observed in simulations for CLIC we observe a 3-ns long high-energy transient followed by a long steady state in Fig. 2. The deceleration process leads to an asymmetric energy distribution shown in Fig. 3 with a significant high-energy tail all the way up to the initial energy in TBL of $150 \mathrm{MeV}$.

The dramatic reduction of beam energy even in TBL permits us to investigate the deteriorated beam quality due to inverse adiabatic damping and the beam becoming less rigid at lower energies. Monitoring the beam quality is therefore vital but very challenging, because the deceleration process also induces a significant energy spread in the beam, which requires spectrometers with large acceptance. Moreover, the energy spread affects the emittance measurements by introducing large chromatic effects in the quadrupoles. Since the beam loading in the PETS is time dependent, it is highly desirable to measure the temporal variation of beam properties along the 140-ns long beam pulse. Finally, the diagnostic devices need to withstand the large beam power and high radiation levels.

In this paper we discuss the diagnostic devices for beamsize and energy measurements in the TBL. For the emittance measurements we utilize optical transition radiation (OTR) screens with adequate light yield and linear over a wide range of beam currents. For the energy measurements we employ large acceptance segmented beam dumps which permit us to diagnose the energy spread along the beam pulse and up to $\pm 18 \%$ energy spread. The main focus is the design, performance, and limitations of these devices.

In the following section we describe the hardware implementation of the diagnostic devices for energy distribution and beam size and emittance. In the subsequent

TABLE I. Beam parameters in the TBL and in the CLIC drivebeam decelerator [1].

\begin{tabular}{lccc}
\hline \hline Parameter & Unit & CLIC & CTF3 \\
\hline Initial energy $\left(E_{\max }\right)$ & $\mathrm{MeV}$ & 2400 & 150 \\
Final energy $\left(E_{\min }\right)$ & $\mathrm{MeV}$ & 240 & 67 \\
Average pulse current & $\mathrm{A}$ & 100 & 28 \\
Pulse train duration & $\mathrm{ns}$ & 240 & 140 \\
Fill time & $\mathrm{ns}$ & 1 & 3 \\
Charge per bunch & $\mathrm{nC}$ & 8.4 & 2.3 \\
Bunch separation & $\mathrm{ps}$ & 83.3 & 83.3 \\
Number of PETS & & 1491 & 16 \\
\hline \hline
\end{tabular}

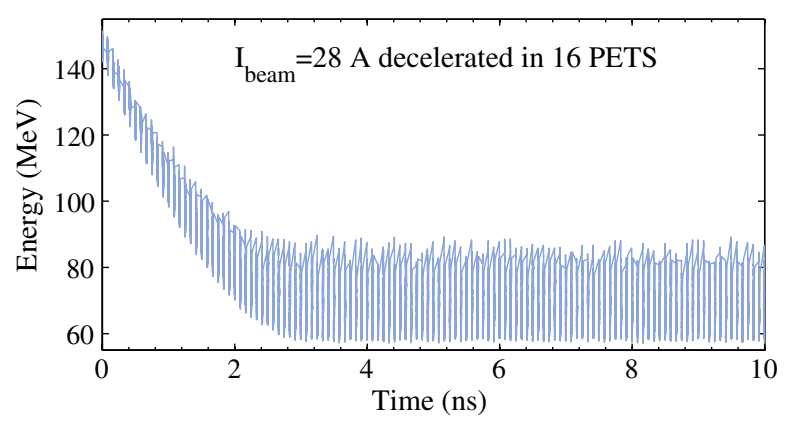

FIG. 2. A PLACET simulation showing the beam energy distribution in TBL during the first $10 \mathrm{~ns}$ of a 28 A beam pulse train, initially at $150 \mathrm{MeV}$, decelerated in 16 PETS. The 3-ns long high-energy transient is followed by a 137 ns steady state with an unusually large energy spread; see Fig. 3.

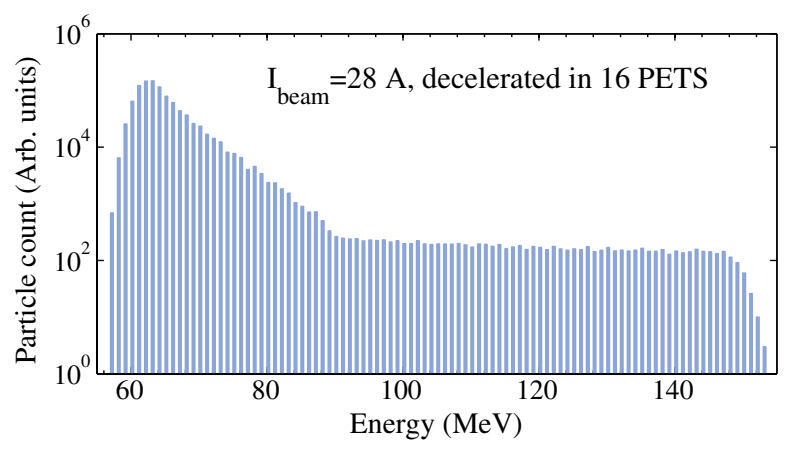

FIG. 3. PLACET simulation of the TBL beam. Histogram of the energy distribution of a 28 A beam decelerated in 16 PETS. The large energy spread is clearly visible, as well as the asymmetric profile, resulting from the high-energy transient.

section we discuss the peculiarities of the OTR radiation and then the use of OTR in emittance measurements of highly chromatic beams. In the next section we discuss the segmented beam dump more closely and compare its performance to what is measured on dispersive OTR screens and with beam dynamics simulations with PLACET. We conclude by discussing the extrapolation of the TBL diagnostics to the CLIC regime.

\section{DIAGNOSTICS IN CTF3}

Transverse beam-size measurements are based on OTR screens observed by a camera. For the time-resolved 
energy measurement we employ a segmented beam dump or a device we coined slit dump.

We first consider the OTR-based diagnostics, which in CTF3 has the following standard layout: a vacuum tank containing the OTR screens and an optical line from the view port of the tank to a CCD camera imaging the light emitted in the backward direction. The screens for transverse profile measurements are employed mostly for determining the beam emittance and Twiss parameters through quadrupole scans. The system is the latest design implemented at CTF3 with a four-position arm supporting a replacement chamber, two screens, and a calibration target. The replacement chamber was implemented to minimize the disturbance to the beam by diagnostic equipment when not in use. The first screen is made of chemical vapor deposition $\mathrm{SiC}$, able to withstand the thermal load of the high-intensity beam [9]. The second one, made of $\mathrm{Si}$, though slightly worse from a thermal perspective, has a higher reflection coefficient and is used at a lower current. Both screens have a $30 \mathrm{~mm}$ diameter, are $200 \mu \mathrm{m}$ thick with a $15^{\circ}$ tilt, and have been polished to mirror quality. The resolution of the transverse profile measurement is $50 \mu \mathrm{m}$, which has been established by simulations in the ZEMAX software [10]; see Sec. III for details. This rather moderate resolution is enough to meet the requirements at CTF3, where the beam sizes are comparatively large. Clearly, the CCD camera has a finite integration time that makes single-shot measurements possible but not intrapulse-train monitoring.

The screen systems for spectrometry accommodate only one fixed aluminum screen, approximately $150 \mathrm{~mm} \times$ $50 \mathrm{~mm}$ surface area, $50 \mu \mathrm{m}$ thick, intercepting the beam path at a $45^{\circ}$ angle. In addition, there is a $50 \mu \mathrm{m}$ carbon foil mounted in front of the screen. The beam goes perpendicularly through the carbon foil, which is there to block synchrotron radiation generated in the dipole magnet [11].

Because the distance between the carbon foil and the aluminum screen is smaller than the near-field limit, we need to consider the possibility of interference between the forward OTR from the carbon foil and the backward OTR from the screen [12]. The electromagnetic fields from both sources are reflected on the aluminum screen in the specular direction towards the camera, and since the fields are generated by the same electron the two sources are correlated, in analogy with Young's double slit experiment. In this case the phase difference between the two sources is given by the difference in propagation time from surface to surface of the electron and the field. The resulting intensity distribution on the image plane is characterized by interference peaks and troughs, the position of which depends on the distance between the foils and of the radiation wavelength. However, since the OTR spectrum is broadband and since the camera integrates over the optical range, the interference pattern is smoothened out. Also, the geometrical distribution and the energy spread of the beam provides a similar smearing effect so that the shape of the final intensity profile remains similar to the case with a single foil. A more careful analysis shows that the total light intensity is increased by roughly a factor 2 .

The TL2 and the TBL spectrometer lines hold diffusive aluminum screens, as indicated in Table II. The choice of diffusive radiators follows from extensive studies at CTF3 on how to mitigate the vignetting effect $[13,14]$. Considering the dispersion and the spatial resolution of $\sim 400 \mu \mathrm{m}$ the resolution on energy is better than $0.2 \%$.

For time-resolved energy spectrometry we use segmented beam dumps [11]. These are passive, robust systems and are well adapted to the high-intensity beams at CTF3. A segmented beam dump uses the same detection principle as a Faraday cup. The incoming particles penetrate a metallic block, generate electromagnetic showers, and are finally completely stopped. Every absorbed charge is detected as a current flowing to ground through a $50 \Omega$ resistance and sampled with a fast analog-to-digital converter (ADC). A horizontal segmentation of the metallic block, combined with individual data acquisition from each segment, provides a horizontal beam profile.

A novel segmented beam dump was optimized especially for the TBL based on extensive Fluktuierende Kaskade (FLUKA) and Flair simulations [15-17]. It was installed in January 2011 and commissioned during summer 2011. See $[18,19]$ for details regarding the design.

The segment assembly consists of 32 tungsten plates, 3 -mm wide and spaced by $1 \mathrm{~mm}$ with insulators in alumina. Tungsten has a high stopping power and can withstand extreme thermal loads. Nonetheless, a water-cooled, multislit collimator is used as a thermal buffer placed in front of the segment assembly. The $100 \mathrm{~mm}$ long collimator absorbs most of the beam power and lets only a small fraction of the incoming particles pass through $400-\mu \mathrm{m}$ wide slits, one in front of each segment. It is made of Inermet [20], a metallic compound with a high tungsten content. The slits, and the segments placed just after, are concentric with the bending center in order to match the angles of the incoming particles.

The time response of the segmented dump is ultimately limited by the capacitance between the plates as the beam charge is absorbed. It has been estimated to be below $0.5 \mathrm{~ns}$ through calculation and measured on a prototype to be $0.3 \mathrm{~ns}$ [18]. In its current configuration the detector has a

TABLE II. Characteristics of the spectrometer lines and its monitors used in the TBL study.

\begin{tabular}{lccc}
\hline \hline Location $^{\mathrm{a}}$ & Device & Dispersion $D_{x}$ & Comment \\
\hline TL2 & OTR screen & $0.50 \mathrm{~m}$ & diffusive \\
TL2 & single-slit dump & $0.81 \mathrm{~m}$ & multishot \\
TBL & OTR screen & $0.22 \mathrm{~m}$ & diffusive \\
TBL & segmented dump & $0.35 \mathrm{~m}$ & single-shot \\
\hline \hline
\end{tabular}

${ }^{\mathrm{a}}$ See TBL layout in Fig. 1. 
time resolution limited by the choice of ADCs, which have a sampling rate of $256 \mathrm{MS} / \mathrm{s}$, and secondly by dispersion in cables. However, the $5 \mathrm{~ns}$ time resolution is sufficient for monitoring the intra-pulse-train energy profile.

Installed in a $22.5^{\circ}$ spectrometer line just upstream from TBL, marked as TL2 in the layout in Fig. 1 and in Table II, is a single-slit dump, which is a simple alternative to a segmented beam dump. As the name suggests, it consists of a single detecting segment behind a collimator with a single slit. The segment is a $100-\mathrm{mm}$ long steel cylinder, sampled at $100 \mathrm{MS} / \mathrm{s}$ by an ADC, and is kept electrically insulated by a $25 \mathrm{~mm}$ macor layer inside a steel support. The single-slit collimator consists of another 100-mm long steel cylinder with a 1-mm wide slit that sets the spatial resolution whereas the time resolution is currently limited by the ADC acquisition rate.

By adjusting the spectrometer magnet current, i.e., changing the dispersion, the beam can be steered across the slit dump which thus provides a multishot measurement of the energy profile with $10 \mathrm{~ns}$ time steps. Evidently, a certain stability of the incoming beam will have to be assumed. It allows for a reference measurement of the energy profile before any deceleration has taken place.

At CTF3 a substantial effort has been put into the development of beam profile instruments adapted to high-intensity beams and the demanding radiation environment. The beam instrumentation in TBL is built on developments done for the CTF3 linac and is concentrated to two diagnostics sectors: one in TL2, just before TBL, and one at the end of TBL, as marked in Fig. 1. Both sectors follow the same pattern, shown in the schematic picture in Fig. 4. It includes an OTR screen for transverse profile measurements, another OTR screen for high resolution spectrometry, and a device for time-resolved spectrometry inside the beam dump. Table II contains information on the spectrometer lines in connection to TBL. The devices installed are listed, together with the dispersion used to

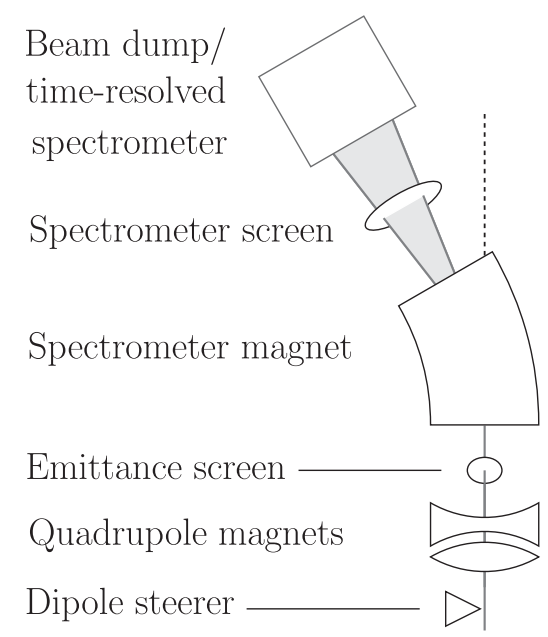

FIG. 4. A schematic layout of a diagnostic sector. deduce the energy spread. Apart from these instruments TBL also holds inductive beam position monitors [21] for beam position and current monitoring, and a streak camera, imaging an OTR screen, for bunch length measurements.

\section{OTR}

We extensively use OTR radiation as beam diagnostics and here we investigate how the imaging is affected by the large energy spread.

OTR is emitted when a charged particle crosses a media interface. The intensity of the emitted light is, although lower than, for example, scintillation light, linear to the number of charge crossings and suffers no risks of saturation. It also has a response time of the order of the electronic relaxation time of the material, and is therefore suitable for imaging high-intensity beams in the transverse as well as the longitudinal dimension.

At ultrarelativistic particle energies and assuming a perfectly conducting radiator, the energy emitted per solid angle and frequency range, in the backward direction, can be simplified to [22]:

$$
\frac{d^{2} W}{d \omega d \Omega}=\frac{q^{2}}{4 \pi^{3} \varepsilon_{0} c} \frac{\theta^{2}}{\left(\gamma^{-2}+\theta^{2}\right)^{2}} .
$$

The angle $\theta$ refers to the angle with the specular direction to the radiator and $\gamma$ is the relativistic Lorentz factor. The emitted energy is in practice independent of wave length in most applications involving electron beams. The angle of maximum emission, $\theta_{\max }=1 / \gamma$, decreases with increasing particle energy so that at energies above a few hundred $\mathrm{MeV}$ the emission pattern is dominated by a cone shape of a few milliradian opening angle.

Integrating Eq. (1) over the radiation frequency and over angles up to a cutoff angle $\theta_{c} \gg \gamma^{-1}$, we obtain an expression for the total number of photons emitted in a frequency range from $\omega_{1}$ to $\omega_{2}$. We see in Eq. (2) [22] that the number of photons grows logarithmically with the particle energy:

$$
N=\frac{\alpha}{\pi}\left[\ln \left(\gamma \theta_{c}\right)^{2}-1\right] \ln \left(\frac{\omega_{2}}{\omega_{1}}\right),
$$

where $\alpha \approx 1 / 137$ is the fine structure constant. This energy dependence constitutes a potential problem for beam imaging using OTR at very large energy spreads and warrants a closer inspection.

We have evaluated the resolution of the OTR systems by performing realistic simulations of the hardware installed, using the physical optics propagation mode [23] of the ZEMAX software [10]. The simulations start from the OTR electric field from Ref. [24] and Ref. [25] (Eq. 8) and propagate the wave front using Fresnel diffraction, taking into account all aberrations and diffraction occurring in the optical line. We show the point spread function (PSF) of a $150 \mathrm{MeV}$ electron for wavelengths $400-600 \mathrm{~nm}$ in Fig. 5. The image plane has been optimized for the 


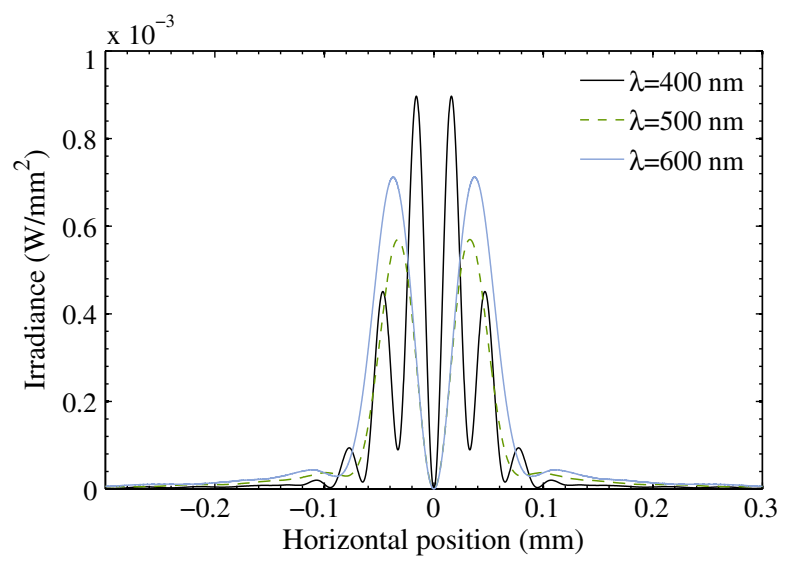

FIG. 5. Vertical cross section of the PSF irradiance at different wavelengths and for $E=150 \mathrm{MeV}$.

wavelength $500 \mathrm{~nm}$, which corresponds to the sensitivity peak of the CCD camera. However, chromatic aberrations will dilute the image. Nonetheless, the rms resolution estimated from the FWHM of the PSF distribution is $44 \mu \mathrm{m}$. The pixel size of the CCD constitutes a resolution limit of $22 \mu \mathrm{m}$, when normalized to the optical demagnification of $M=1 / 6.6$. By adding these two contributions in quadrature, we obtain a total resolution of $50 \mu \mathrm{m}$.

In order to estimate the effect of large energy spreads on the measured beam profiles, we repeated the simulation with varying beam energy while keeping the wavelength fixed at $500 \mathrm{~nm}$. The FWHM of the resulting PSF, shown normalized in Fig. 6, stays remarkably unaffected. Only tails begin to grow for lower energy. For the remainder of this paper we assume a constant PSF for all beam energies. We conclude that OTR is reliable for beam-size measurements in nondispersive sections even for the large energy spread expected at the end of TBL.

Now, we turn our attention to dispersive sections, where there is an obvious correlation between position

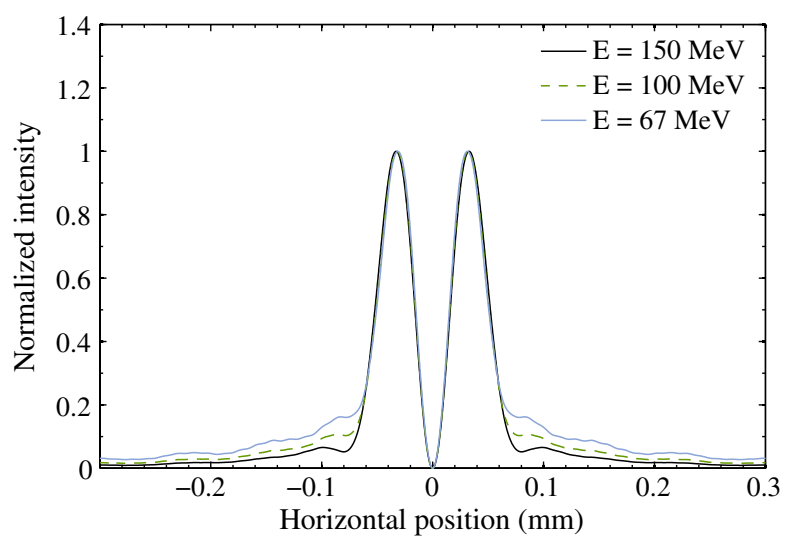

FIG. 6. Vertical cross section of the PSF irradiance (normalized intensity) at beam energies relevant to TBL and for $\lambda=500 \mathrm{~nm}$. on the screen and the amount of light generated. First, we assume a dispersion of $D=0.2 \mathrm{~m}$, similar to the dispersion at the location of the spectrometer screen in TBL. Second, we assume that for a given particle energy a constant light intensity is collected by the imager irrespective of the emission point on the radiator. This is true when there is no or negligible vignetting, which we can achieve by using diffuse radiators and large aperture lenses. In addition, we assume that the light collection efficiency is energy independent, which is supported by our ZEMAX simulations. Then, we utilize Eq. (2) for the correlation between number of photons and the particle energy. Finally, we consider an ideal optical line with a single lens, i.e., point-to-point imaging, in which case the angular distribution of the emitted light can be completely ignored. In order to avoid diffraction, which is likely when the OTR angular distribution is wide, one should choose large aperture lenses, as is done at CTF3.

We let the energy profile of a decelerated beam generated in PLACET, like the one in Fig. 3, act as the reference distribution. The peak energy has been used as a reference energy and OTR intensity and the OTR distribution has been calculated relative to that reference. Figure 7 shows the result where the black dashed line represents the particle density as a function of energy while the green line corresponds to the light density profile. As should be clear from the figure, the discrepancy is barely visible, except for in the high-energy tail. This will be true as long as the optical aperture remains large. For smaller aperture the discrepancy grows in the high-energy tail. In practice it is negligible compared to other phenomena that limit the accuracy of the spectrometer measurements, such as beam stability.

We find that in the parameter range relevant for TBL the energy dependence of the emission of OTR radiation does not play a role.

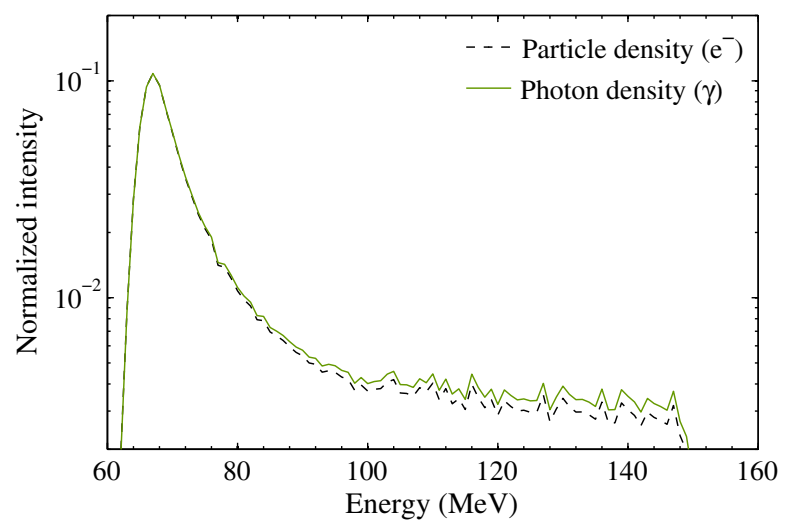

FIG. 7. The energy distribution of a decelerated drive beam: the initial particle distribution compared to the intensity distribution of the collected OTR. The difference is visible only in the high-energy tail. 


\section{CHROMATICITY IN QUADRUPOLE SCANS}

As already mentioned the emittance and the Twiss parameters are measured in TBL through quadrupole scans and subsequent beam-size measurements on OTR screens. In quadrupole scans the strength of the quadrupole is varied and consequently the chromatic effects in the quadrupoles are changed, leading to an effect of the energy spread on the measured spot size. A large energy spread will therefore, if uncorrected, lead to a misinterpretation of the emittance.

In Ref. [26] this effect was addressed in a perturbative way, which is adequate in the case of a Gaussian energy distribution with a moderate rms spread of a few percent, but in the CLIC drive-beam decelerator and even in the TBL, the energy spread can easily exceed tens of percent. Moreover, the profile is vastly different from Gaussian, which warrants a careful analysis, of both the nonperturbative regime as well as non-Gaussian beams.

In [7] we have carefully studied this effect through a more general approach. We there considered a thin-lens model with two quadrupoles used for the quadrupole scan emittance measurement setup, where the second quadrupole is kept at fixed strength while the focusing strength of the first is varied. Here, we extend the problem further to include thick lenses. The beam size on the screen for a given beam line configuration, as a function of the incoming beam parameters $\sigma_{i j}$ that we want to determine, is given by

$\bar{\sigma}^{2}\left(k_{i}\right)=R_{11}^{2}\left(k_{i}\right) \sigma_{11}+2 R_{11}\left(k_{i}\right) R_{12}\left(k_{i}\right) \sigma_{12}+R_{12}^{2}\left(k_{i}\right) \sigma_{22}$,

where $R$ is the transfer matrix, the elements of which depend on the quadrupole setting. In a quadrupole scan measurement the beam-size $\bar{\sigma}$ is recorded for a sequence of quadrupole settings $k_{i}$. The result is a linear equation system consisting of copies of Eq. (3), which needs to be inverted in order to determine the incoming beam parameters. As in Ref. [7] we use the fact that the quadrupole strength $k$ depends on the momentum and let $k(\delta)=$ $k_{0} /(1+\delta)$, with $k_{0}$ being the strength corresponding to an on-momentum particle. In the thick-lens approach the expression for $R$ becomes long and cumbersome and we treat the equation by numerically determining the matrix elements. The effect of energy spread is taken into account in a consistent way through summation over all momenta, weighted by the momentum density function $\psi(\delta)$, through integrals of the type

$$
R_{11}^{2}\left(k_{0}\right)=\int \psi(\delta) R_{11}^{2}\left(\frac{k_{0}}{1+\delta}\right) d \delta,
$$

and similar expressions for $R_{11} R_{12}$ and $R_{12}^{2}$.

In order to assess the severity of the effect we consider the nominal TBL beam that has suffered an energy loss of $55 \%$ and has the energy distribution shown in Fig. 3 as $\psi$ to

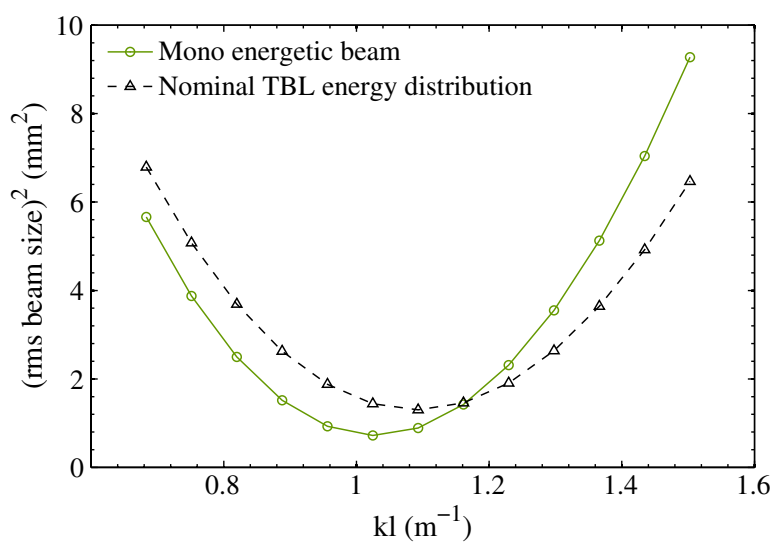

FIG. 8. The beam size changes differently during a quadrupole scan depending on the energy spread in the beam.

evaluate the matrix elements $R_{i j}(k)$. We then show the dependence of the beam size on the screen $\bar{\sigma}$ squared as a function of the excitation of the first quadrupole in Fig. 8. For comparison we also show the beam size for a monochromatic beam. We see that the two parabolas differ significantly. The waist has moved indicating that the Twiss parameter $\alpha$ is different. The minimum is also different, indicating that the beam size is affected. Finally, the wings of the parabola for the monochromatic curve are much steeper, which indicates that the angular divergence is affected. Conversely, if we would use the monochromatic model to determine the Twiss parameter from data that stem from a TBL beam, we would introduce significant systematic errors. For the data shown in Fig. 8 we find that we overestimate the emittance by $20 \%$, while we underestimate the beta function by $34 \%$ and alpha by $6 \%$.

In addition, we analyzed data from emittance measurements in TBL with only 13 PETS installed. We consider the measured energy profile as $\psi$ to recalculate the transfer matrix $R$ for every quadrupole setting and invert the matrix system in Eq. (3). We thus obtain new Twiss parameters, presented in Table III, for which the large energy spread has been taken into account. In this case the discrepancy between the result obtained with the corrected algorithm and the conventional monochromatic model is $6 \%$ for the emittance, $11 \%$ for the beta function, and $4 \%$ for alpha.

Fortunately, the correction will be fairly straightforward. If we know or can measure the energy distribution, we can

TABLE III. Results from a quadrupole scan measurement analyzed with the fully chromatic, thick-lens model compared to the monochromatic model.

\begin{tabular}{lccc}
\hline \hline Model & $\varepsilon_{N}$ & $\beta$ & $\alpha$ \\
\hline Monochromatic & $350 \mathrm{~mm} \mathrm{mrad}$ & $12.2 \mathrm{~m}$ & -3.0 \\
Chromatic & $330 \mathrm{~mm} \mathrm{mrad}$ & $13.6 \mathrm{~m}$ & -3.2 \\
Deviation & $+6 \%$ & $-10 \%$ & $-4 \%$ \\
\hline \hline
\end{tabular}


numerically evaluate the influence of the energy distribution on the transfer matrix and adapt the equations that relate the measured beam sizes and the initial beam parameters [Eq. (3)] before performing the linear fit, which takes the systematic effect due to the large energy spread into account comprehensively.

We have shown that the OTR based profile monitors faithfully reproduce the profile of the highly decelerated beam. In the following section we focus on the timeresolved energy measurement.

\section{SEGMENTED DUMP PERFORMANCE}

The performance of the segmented beam dump has been investigated both through simulations and through beambased measurements. A description of the procedures and results follows in this section.

The segment width and spacing were optimized for containing both primary and secondary particles within the segment. Nonetheless, there will always be a certain level of crosstalk because of scattered particles. The effect of this crosstalk on the detector resolution has been investigated using FLUKA. By letting a $150 \mathrm{MeV}$ electron beam impinge on the middle segment and studying the signal leakage in the form of scattered particles, the lower resolution limit can be obtained. The rms width of a Gaussian fit to the profile obtained through this simulation was extracted. This width, $\sigma_{\text {part }}=2.71 \mathrm{~mm}$, is interpreted as the minimum reproducible beam width.

Another effect that is expected to broaden the measured beam profile is the presence of thin foils in the spectrometer line, such as the $50 \mu \mathrm{m}$ aluminum screen for OTR generation, and $50 \mu \mathrm{m}$ carbon foil, as described in Sec. II. Additionally, there is a $100 \mu \mathrm{m}$ aluminum vacuum window just upstream from the detector. Together, these foils are expected to increase the beam divergence by $3 \mathrm{mrad}$, equivalent to a minimum $\mathrm{rms}$ beam width of $\sigma_{\text {scatt }}=1.71 \mathrm{~mm}$ at the position of the dump.

Considering these two main effects that will smear the beam profile before reconstruction, a lower resolution limit $\sigma_{\text {res }}$ is obtained by adding the corresponding beam widths in quadrature in the following manner:

$$
\sigma_{\text {res }} \geq \sqrt{\sigma_{\text {part }}^{2}+\sigma_{\text {scatt }}^{2}}=3.2 \mathrm{~mm} \Rightarrow \frac{\sigma_{E}}{E} \geq 0.9 \% .
$$

A more extensive investigation of the effect of particle crosstalk on measured beam size is presented in Fig. 9. Gaussian beams of increasing widths have been used as input to FLUKA simulations and the profile obtained with the segmented dump has been fitted to a Gaussian. The rms width of the reconstructed profile is here shown as a function of the input beam size. Once the rms width of the input Gaussian beam is $>7 \mathrm{~mm}$ the overestimation from the segmented dump is less than $10 \%$. The result of these simulations can be used to correct the measurement profile widths. Also, the broadening from scattering in foils

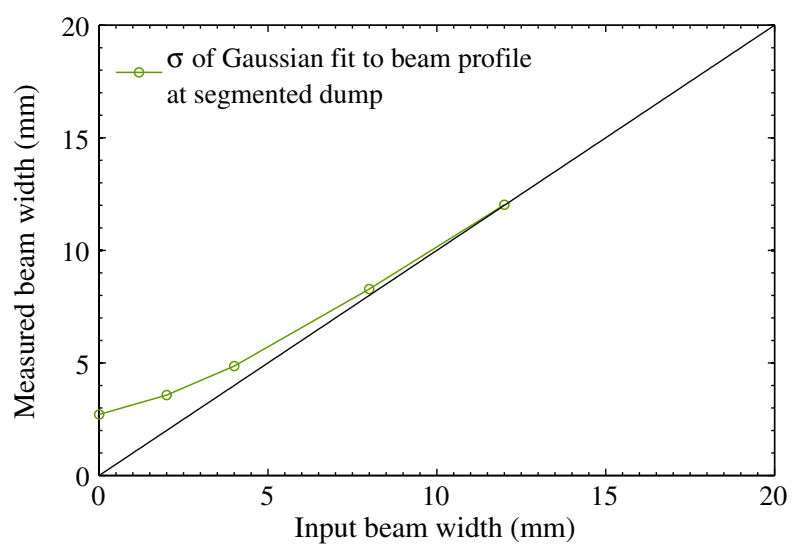

FIG. 9. FLUKA simulations: width of reconstructed distribution as a function of the width of the input beam.

can be estimated with FLUKA simulations for different beam energies and also used as an adjustment to the measurement.

The expected accuracy of the measurement was investigated with FLUKA. The final detector geometry was used, together with beam distributions obtained with PLACET. As is shown in Fig. 10, the segmented dump reproduces the asymmetric beam profile well. For 4 PETS the FWHM beam size obtained with FLUKA is $13 \%$ larger than the reference beam profile. For 8 PETS, the equivalent value is $7 \%$, and reaches $4 \%$ for 16 PETS. In all cases the discrepancy is reduced to below $2 \%$ by subtracting in quadrature the broadening expected from segment crosstalk and the intrinsic beam size.

The performance of the segmented dump has been tested through a series of measurements. The response of individual segments and the alignment of the system has been tested using a dipole scanning technique, in which the beam is steered across the detector in small steps.

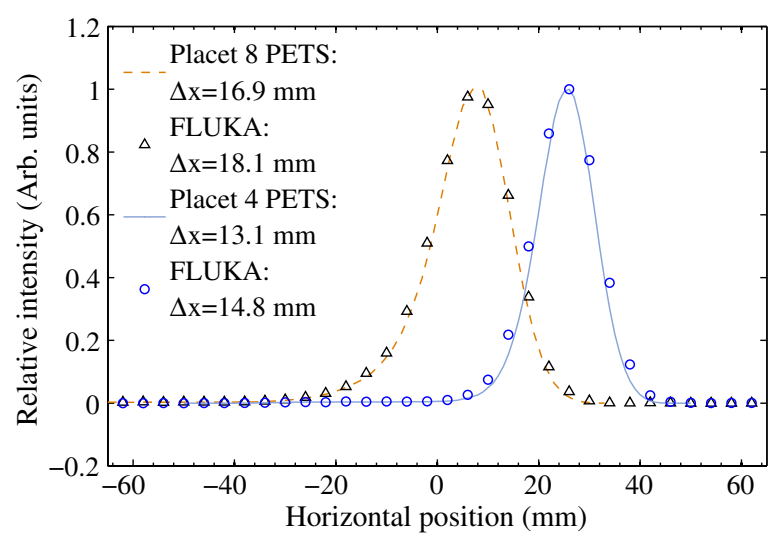

FIG. 10. FLUKA simulations of the detector performance on profile reconstruction. Expected beam distributions at the position of the segmented dump for 4 and 8 PETS, obtained with PLACET, has been used as input. The width $\Delta x$ refers to the full width at half maximum. 
Figure 11 shows the result of such a measurement. During a dipole scan each segment is used separately to sample the beam. The resulting spectra are integrated over a selected time window, thus providing a beam profile as a function of dipole current—one profile for every segment. The peak of this profile is used as the segment response.

The segmented dump in TBL gives a fairly uniform response, much thanks to the concentric slit and segment geometry. The response curve is well reproducible, illustrated in Fig. 11 with statistical error bars calculated from a series of dipole scan measurements; although, a 100-mm wide vacuum chamber upstream from the detector currently reduces the response drastically of three channels on each side, as commented on in Ref. [18]. Small additional response variations from segment to segment are believed to arise from slit width variations and from a minor misalignment of the segment with respect to the slit.

The OTR screen in the spectrometer line is used as a reference for comparing measurements to the new device. The intrinsic beam size has been subtracted from both measurements and the segmented dump profile has been corrected for particle crosstalk and scattering in foils. Figure 12 shows two single-shot measurements from the segmented dump and the OTR screen, one where the energy spread is large and one with smaller energy spread, corresponding to high and low beam current, respectively. Another example of where the segmented beam dump has been used for different beam conditions are presented in Figs. 25 and 26 in Ref. [18]. In cases of large energy spreads, like the one presented here and in Fig. 26 in Ref. [18], the agreement stays within the shot-to-shot accuracy of each measurement. At small energy spreads, also in Fig. 12, the segmented dump measures a 4\% larger energy spread, most likely due to its limited granularity and resolution. For small energy spreads, the intrinsic beam size remains a more significant fraction of the

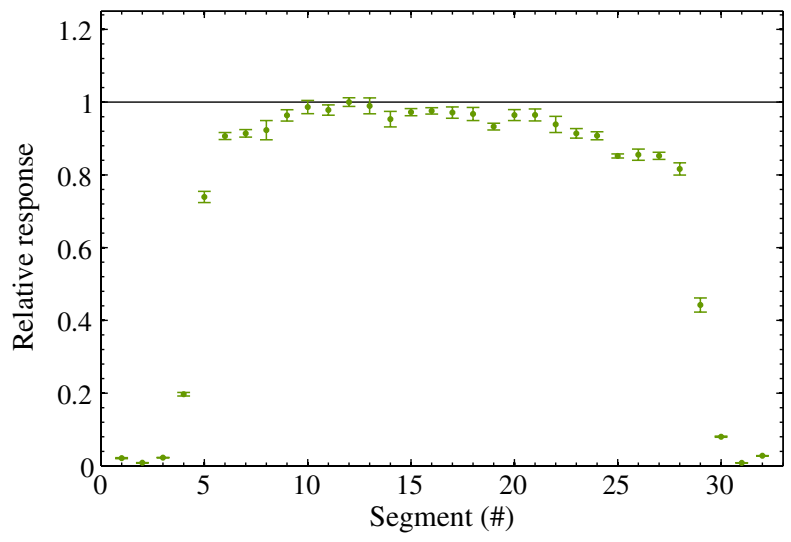

FIG. 11. Response of individual segments. The response is calculated by scanning the beam over each segment separately, then taking the maximum of the integrated profile. This plot is a refined analysis of data presented in Fig. 24 in Ref. [18].

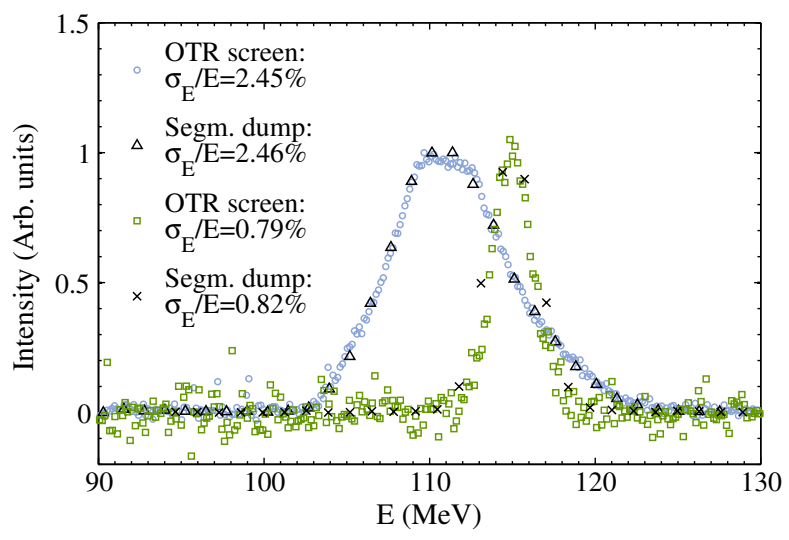

FIG. 12. Comparison of the segmented dump with the OTR screen. After applying corrections to the measurement, the segmented dump measurement gives an up to $4 \%$ overestimation compared to the OTR screen. For larger beams the discrepancy is negligible.

measured beam size. The uncertainty of the measurement is thereby larger in this case. Since the resolution of the OTR screen is far superior to that of the segmented dump and because the OTR system is well understood, we trust the screen measurement to be correct. Based on this we conclude that the resolution of the segmented dump is $1 \%$. This corresponds well to the value expected from simulations; refer to the discussion above.

The energy spectrum as measured with the segmented dump has also been compared to what is expected from the model implemented in PLACET. Apart from energy measurements, the beam is monitored in several ways during TBL operation: beam position monitors for beam intensity and position, streak camera for bunch length measurements; OTR screens for beam-size measurements and for extracting the Twiss parameters from quadrupole scans. The result of the latter is used for beam-size adjustment in spectrometry measurements and the incoming beam parameters (current, bunch length, emittances, energy, and energy spread) are then used as input to PLACET simulations. Thus, the predicted particle distribution at the end of the line can be obtained and compared with the beam profile as measured with the segmented dump.

Several beam parameters, such as beam intensity, trajectory, or phase of individual bunches, may vary along the pulse train and thus influence the deceleration process. This leads to variations along the pulse in the final energy spectrum. A slice in time is therefore selected from the energy spectrum in order to minimize these influences where possible.

Figure 13(a) shows a typical beam energy spectrum as measured with the single-slit dump in TL2. The incoming pulse train has an energy of $125 \mathrm{MeV}$ and a $0.5 \% \mathrm{rms}$ energy spread. The corresponding energy spectrum in TBL, as measured with the segmented dump, is presented in Fig. 13(b). Here, the mean energy is decreased to 

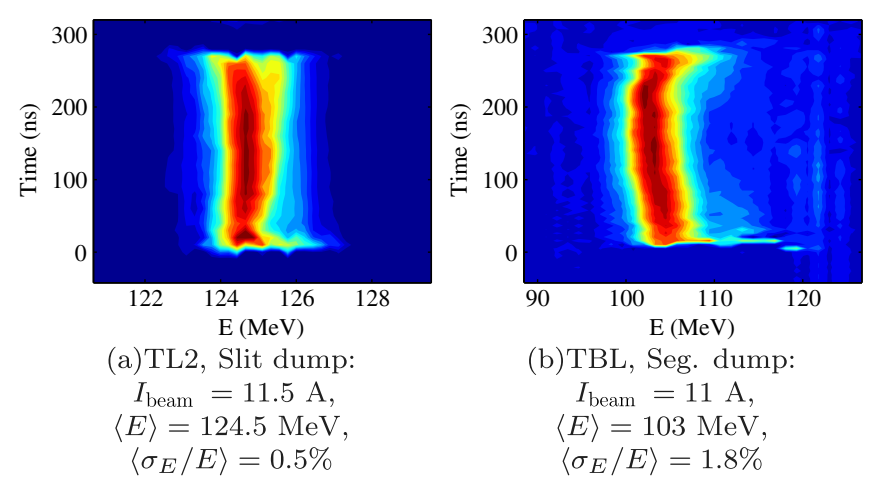

(b)TBL, Seg. dump:

$I_{\text {beam }}=11 \mathrm{~A}$,

$\langle E\rangle=103 \mathrm{MeV}$

$\left\langle\sigma_{E} / E\right\rangle=1.8 \%$

FIG. 13. Beam energy spectrum before entering TBL (a) and after the deceleration in 13 PETS (b). The first bunch arrives at time $t=0$. Note that the spectrum to the left is acquired over many pulse trains while the spectrum to the right is a single-shot measurement.

$103 \mathrm{MeV}$, i.e., $22 \mathrm{MeV}$ deceleration. The rms energy spread is more than tripled to $1.8 \%$.

In Fig. 14 we show an example of the measured particle distribution compared to the distribution obtained with PLACET. Here, the energy parameters in Fig. 13(a) have been used as input to PLACET and an approximately $50 \mathrm{~ns}$ window with the minimum energy was chosen from the spectrum in Fig. 13(b). The measured distribution has a FWHM width of $19 \mathrm{~mm}$ while the PLACET distribution has an equivalent width of $20 \mathrm{~mm}$. After adjusting the measured profile with the intrinsic beam size and known profile broadening effects we obtain a final energy spread of $4.3 \%$ (FWHM). This is very close to the value of $4.2 \%$ extracted from the PLACET simulation. Therefore, the measured profile agrees within the measurement accuracy with what is expected from simulation, both with respect to the peak energy, the shape of the profile, and the FWHM energy spread.

In Fig. 15 we show the energy extracted from the beam determined by three methods. First we consider the rf

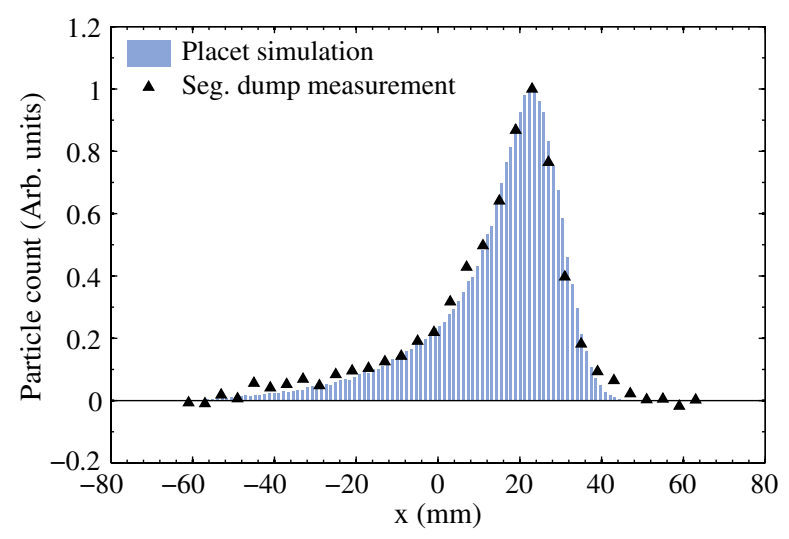

FIG. 14. The measured beam profile after deceleration in 13 PETS compared to the profile expected from PLACET simulations.

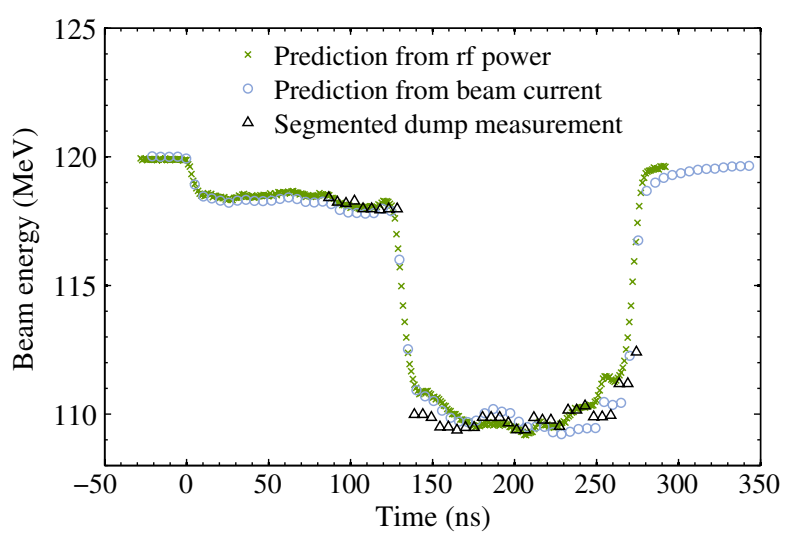

FIG. 15. The measured energy along the pulse train after deceleration in 4 PETS. The prediction from the extracted $\mathrm{rf}$ power and from the beam current measurements are shown for comparison. Incoming beam: $I_{\text {beam }}=18 \mathrm{~A}, E=120 \mathrm{MeV}$.

pulse as measured in the PETS and calculate the corresponding energy loss of the beam. Second, we calculate the expected energy from the longitudinal intensity profile of the pulse together with the charge distribution form factor. Third, we extract the average beam energy along the pulse from the measurement done with the segmented beam dump. The agreement is very good and the segmented dump measurement indicates similar temporal variations as those seen in the rf signals. Reference [27] contains a more detailed analysis of these measurements. We conclude that the segmented dump provides reliable time-resolved energy measurements that agree perfectly well with the OTR screen measurements, with the energy loss predicted from the extracted rf power and with what is expected from PLACET simulations.

\section{EXTRAPOLATING TO CLIC}

As an outlook we here discuss the extrapolation of the presented diagnostic techniques to the beam parameters of the CLIC drive-beam decelerator, listed in Table I.

Emittance measurements based on quadrupole scans or multiple screen use will be affected by chromatic effects in the CLIC decelerator lattice due to the even larger energy spread than in the TBL. Nevertheless, the techniques can be used reliably if the energy spread is taken care of by correcting the measurement analysis as described in Ref. [7].

Beam imaging with OTR screens is an accurate method for high charge beams, though radiator materials must be chosen with great care. In the CLIC drive beam there is a continued need for large aperture lenses in order to avoid diffraction and vignetting and to minimize the difference in light collection between the most and the least decelerated particle.

In spectrometer lines the profile measurement using OTR screens is expected to remain sufficiently reliable. The calculation presented in Fig. 7 was made also for the 
CLIC energy distribution and the FWHM width of the OTR profile differs from the particle profile by less than 5\%. The largest difference between particle distribution and OTR distribution occurs in the high-energy tail. Conveniently, a calibration curve can be generated by combining Eq. (2) with the dispersion on the screen in order to compensate for the lower light yield at lower energy.

Because of the high thermal load imposed by the beam, segmented beam dumps will not be applicable to the CLIC drive beam. It is first of all the mechanical stress induced by repetitive beam shots that will cause a problem for this particular technique, see [18], and another method will have to be developed.

\section{CONCLUSION}

The rf distribution scheme for CLIC relies on the deceleration of a high-intensity drive beam. The power extraction from the drive beam deteriorates the beam quality and increases the energy spread significantly, which calls for careful monitoring of the beam properties. This process is being addressed experimentally in TBL at the CLIC Test Facility, where several beam monitors are present to supervise the decelerated beam. In this paper we have investigated the performance of the beam profile monitors in TBL. In particular, we have investigated the influence of the large energy spread on the quality of the diagnostic methods, with the final prospect of implementation in the CLIC decelerator.

The large energy spread induces significant chromatic effects when emittance is measured through quadrupole scans or multiple screens. Yet, the methods are still reliable seeing that we have described a way to correct for it.

OTR is an excellent technique for beam imaging due to its charge linearity. Using OTR screens the beam profile is faithfully reproduced also for beams with large energy spread, both in absence and presence of dispersion.

Time-resolved energy measurements are done successfully with segmented beam dumps in a spectrometer line. The detector performance has been fully characterized and its limitations well understood.

We have comprehensive diagnostics for monitoring the highly decelerated beam in the TBL. All the measurements are in coherence and in agreement with simulations.

\section{ACKNOWLEDGMENTS}

We wish to acknowledge the support of the CTF3 operation team for its everyday help and support during TBL operation. Thanks also to the CTF3 Collaboration for support and construction of TBL. Author M. Olvegård is supported by the EU under Contract No. PITN-GA-2008215080 .
[1] H. Braun et al., CERN Report No. CLIC-Note-764, 2008.

[2] I. Syratchev, D. Schulte, and E. Adli, in Proceedings of the 22nd Particle Accelerator Conference, Albuquerque, USA, 2007 (IEEE, New York, 2007), p. 2194.

[3] E. Adli, Ph.D. thesis, University of Oslo, 2009.

[4] E. Adli, R. Ruber, V. Ziemann, R. Corsini, A. Dubrovskiy, and I. Syratchev, Phys. Rev. ST Accel. Beams 14, 081001 (2011).

[5] D. Schulte et al., CERN Report No. CERN-PS-2000-028$\mathrm{AE}, 2000$.

[6] The tracking code PLACET, https://savannah.cern.ch/ projects/placet/, 2010.

[7] M. Olvegård and V. Ziemann, Nucl. Instrum. Methods Phys. Res., Sect. A 707, 114 (2013).

[8] G. Geschonke et al. (The CLIC Study Team), CTF3 Design Report No. CERN/PS-2002-008 (RF), 2002.

[9] E. Bravin, T. Lefèvre, and C. Vermare, CERN AB BDI Report No. 050, 2003.

[10] The ZEMAX software, version 12 R2 SP1 EE, October 15, 2012, from Radiant Zemax LLC, The Granary, Crowhill Farm, Ravensden Road, Bedford, MK44 2QS, United Kingdom.

[11] T. Lefèvre et al., in Proceedings of the 10th European Particle Accelerator Conference, Edinburgh, Scotland, 2006, (EPS-AG, Edinburgh, Scotland, 2006), p. 1205.

[12] L. Wartski, S. Roland, J. Lasalle, and G Filippi, J. Appl. Phys. 46, 3644 (1975).

[13] C. P. Welsch, E. Bravin, and T. Lefèvre, Rev. Adv. Mater. Sci. 16, 73 (2007) [http://www.ipme.ru/e-journals/RAMS/ no_11607/welsch.pdf].

[14] M. Olvegård et al., in Proceedings of the 10th European Workshop on Beam Diagnostics and Instrumentation for Particle Accelerators, Hamburg, Germany, 2011, p. 413.

[15] G. Battistoni, F. Cerutti, A. Fasso, A. Ferrari, S. Muraro, J. Ranft, S. Roesler, and P. R. Sala, AIP Conf. Proc. 896, 31 (2007).

[16] A. Fasso et al., CERN Report No. CERN-2005-10; INFN Report No. INFN/TC_05/11; SLAC Report No. SLAC-R773, 2005.

[17] V. Vlachoudis, in Proceedings of the International Conference on Mathematics, Computational Methods \& Reactor Physics (M\&C 2009), Saratoga Springs, New York, 2009, (American Nuclear Society, LaGrange Park, IL, 2009).

[18] M. Olvegård et al., Nucl. Instrum. Methods Phys. Res., Sect. A 683, 29 (2012).

[19] M. Olvegård et al., in Proceedings of the 1st International Particle Accelerator Conference, Kyoto, Japan, 2010 (ICR, Kyoto, 2010), p. 1113.

[20] Densimet and Inermet tungsten alloys, http:// www.plansee.com/en/Materials-Tungsten-403.htm, 2010.

[21] J. J. García-Garrigós et al., in Proceedings of the 10th European Workshop on Beam Diagnostics and Instrumentation for Particle Accelerators, Hamburg, Germany, 2011 (DESY, Hamburg, 2011), p. 335.

[22] K. Honkavaara, Ph.D. thesis, Helsinki Institute of Physics; Report No. HIP-1999-04, 1999. 
[23] A. Locke, http://www.radiantzemax.com/kb-en/ Knowledgebase/Exploring-Physical-Optics-Propagationin-Zemax.

[24] M. Castellano and V. A. Verzilov, Phys. Rev. ST Accel. Beams 1, 062801 (1998).

[25] D. Xiang and W.-H. Huang, Nucl. Instrum. Methods Phys. Res., Sect. A 570, 357 (2007).
[26] A. Mostacci, M. Bellaveglia, E. Chiadroni, A. Cianchi, M. Ferrario, D. Filippetto, G. Gatti, and C. Ronsivalle, Phys. Rev. ST Accel. Beams 15, 082802 (2012).

[27] R. L. Lillestøl, S. Döbert, M. Olvegård, A. Rabiller, G. Sterbini, and E. Adli, in Proceedings of the 3rd International Particle Accelerator Conference, New Orleans, Louisiana, 2012 (IEEE, Piscataway, NJ, 2012), p. 1885. 